\title{
Softening of Zr-based metallic glass induced by nanosecond pulsed laser irradiation
}

\author{
Hu Huang a, b, *, Minqiang Jiang ${ }^{\text {c, d }}$, Jiwang Yan ${ }^{\text {b, ** }}$ \\ a School of Mechanical Science and Engineering, Jilin University, Changchun, Jilin, 130022, PR China \\ b Department of Mechanical Engineering, Faculty of Science and Technology, Keio University, Yokohama, 223-8522, Japan \\ c State Key Laboratory of Nonlinear Mechanics, Institute of Mechanics, Chinese Academy of Sciences, Beijing, 100190, PR China \\ d School of Engineering Science, University of Chinese Academy of Sciences, Beijing, 100049, PR China
}

\section{A R T I C L E I N F O}

\section{Article history:}

Received 21 February 2018

Received in revised form

23 April 2018

Accepted 24 April 2018

Available online 27 April 2018

\section{Keywords:}

Metallic glass

Laser irradiation

Nanoindentation

Softening

Serrated flow

Shear band

\begin{abstract}
A B S T R A C T
Laser irradiation has been recently used to tune the mechanical properties of metallic glasses (MGs). However, the mechanism for plasticity improvement by laser irradiation is still not completely clear and requires further investigation. In this study, nanosecond pulsed laser irradiation is applied on a Zr-based MG surface under various laser powers, scanning speeds, and numbers of irradiation cycles. Then, the mechanical properties of the as-cast and laser-irradiated MG surfaces are characterized by nanoindentation tests. In particular, the indentation hardness, load-depth curve, serrated flow, and residual indent morphology of the surfaces are compared. The results show that the nanosecond pulsed laser irradiation softens the irradiated regions; the effect of the irradiation is slightly dependent on the laser irradiation parameters, and especially the laser scanning speed. Furthermore, laser irradiation considerably affects serrated flows in the load-depth curve and shear bands on the top surface, denoting different plastic deformation characteristics compared with the non-irradiated sample. These differences could be rationalized in terms of laser irradiation-induced formation of pre-existing shear bands in the subsurface layer, as well as thermal effects. The present results are expected to enhance the general understanding of the effects of laser irradiation on the mechanical properties of MGs.
\end{abstract}

(C) 2018 Elsevier B.V. All rights reserved.

\section{Introduction}

Metallic glasses (MGs), formed by very fast cooling of molten alloys, possess a long-range disordered atomic structure. This amorphous structure imparts several unique mechanical, chemical, and physical properties to the MGs compared with their crystalline counterparts, such as higher strength, hardness, elastic limit, and wear/corrosion resistance. However, MGs tend to suffer catastrophic failures with very limited tensile plasticity due to rapid propagation of shear bands, which hugely hinders their wider practical applications as structural and functional materials.

Under applied tensile stress, a dominant shear band uninhibitedly propagates in MGs and rapidly reaches a critical shear displacement, leading to crack initiation. However, other shear

\footnotetext{
* Corresponding author. School of Mechanical Science and Engineering, Jilin University, Changchun, Jilin, 130022, PR China.

** Corresponding author.

E-mail addresses: huanghu@jlu.edu.cn (H. Huang), yan@mech.keio.ac.jp (J. Yan).
}

bands only bear a relatively small strain. Hence, a basic approach to improve the plasticity of MGs is to distribute the applied strain over more shear bands, i.e., stimulating multiple shear bands, which limit the propagation of individual shear bands [1-3]. Accordingly, several methods have been proposed to improve the plasticity of MGs, such as introducing nanocrystalline and second-phase particles into the amorphous matrix [4-7] or tuning the residual stress and free volume by pre-deformation [1,8-11].

Recently, laser irradiation was introduced as a way to tune the mechanical properties of MGs in order to achieve enhanced plasticity. Using laser surface melting (LSM, involving direct laser irradiation of a MG surface), plasticity improvement was reported for various kinds of MGs, such as $\mathrm{Zr}_{55} \mathrm{Cu}_{30} \mathrm{Al}_{10} \mathrm{Ni}_{5}$ [12], $\left(\mathrm{Zr}_{0.55} \mathrm{Al}_{0.1-}\right.$ $\left.\mathrm{Ni}_{0.05} \mathrm{Cu}_{0.3}\right)_{99} \mathrm{Y}_{1}$ [13], $\quad \mathrm{Cu}_{46.5} \mathrm{Zr}_{47.5} \mathrm{Al}_{5} \mathrm{Co}_{1} \quad$ [7], $\mathrm{Zr}_{41.2} \mathrm{Ti}_{13.8} \mathrm{Cu}_{12.5-}$ $\mathrm{Ni}_{10} \mathrm{Be}_{22.5}$ (Vitreloy 1) [14], and so on. For example, Chen et al. [12] reported that, compared with the as-cast $\mathrm{Zr}_{55} \mathrm{Cu}_{30} \mathrm{Al}_{10} \mathrm{Ni}_{5} \mathrm{MG}$, which did not exhibit distinct plasticity, the corresponding LSMtreated MG showed a compressive plastic strain of 5.3\% prior to fracture. Plasticity improvement was also achieved for various MGs using laser shock peening (laser irradiation with an absorption 
layer on the MG surface) [15-20]. The enhanced plasticity of irradiated MGs was generally ascribed to laser irradiation-induced complex residual stresses, increase in free volume, formation of pre-existing shear bands or crystalline phases, and structural heterogeneity, as well as to combinations of these effects. Although many previous studies have explored the mechanism for plasticity improvement by laser irradiation, the specific origin of this effect is still not clear and further investigations are required. As plastic deformation in MGs is related to the formation of localized shear bands possessing micro/nanoscale features, nanoindentation with relatively high displacement, load, and time resolution has emerged as a potential technique to investigate plastic deformation in MGs [21-25]. By nanoindentation, the changes in mechanical properties induced by various processing methods can be characterized. Furthermore, by the combined analysis of load-depth curves and residual indent morphologies, plastic deformation in MGs could be further understood. In this study, the nanoindentation method was used to investigate the mechanical behavior of a Zr-based MG surface irradiated with a nanosecond pulsed laser. The indentation hardness, load-depth curve, serrated flow, and residual indent morphology of the as-cast and laserirradiated MG surfaces were analyzed and compared.

\section{Materials and methods}

A $\mathrm{Zr}_{41.2} \mathrm{Ti}_{13.8} \mathrm{Cu}_{12.5} \mathrm{Ni}_{10} \mathrm{Be}_{22.5} \mathrm{MG}$ (Vitreloy 1 ) slice with diameter of $10 \mathrm{~mm}$ and thickness of $1 \mathrm{~mm}$ was obtained from an as-cast MG rod by wire electrical discharge machining (wire-EDM). To obtain a smooth surface and at the same time remove the crystallization layers formed during EDM [26,27], the MG sample was ground using 400, 800, and 1500 grit sand papers in sequence, then polished by diamond abrasive paste and cleaned with alcohol. Previous research $[28,29]$ indicated that during laser irradiation $\mathrm{Zr}$ based MGs react with nitrogen gas but not with argon gas; hence, argon gas at a pressure of $0.05 \mathrm{MPa}$ was used as the shield gas. A Nd:YAG nanosecond pulsed laser system (LR-SHG, MegaOpto Co., Ltd., Japan) with a wavelength of $532 \mathrm{~nm}$ and a pulse width of 15.4 ns was used to irradiate the MG sample. The laser beam was shaped into a spot with a diameter of $\sim 85 \mu \mathrm{m}$. The pulse frequency was controlled to $1 \mathrm{kHz}$. Fig. 1 illustrates the laser irradiation path: line irradiation started from the left side and ended on the right side, and the total length of one irradiated line was $2 \mathrm{~mm}$. After one line irradiation, the laser beam was shifted by $45 \mu \mathrm{m}$ along the feed direction, and thus the overlap width between two irradiation lines was $40 \mu \mathrm{m}$. For comparison, tests with different laser scanning speeds, average laser powers, and numbers of irradiation cycles were performed on the same sample, corresponding to the five

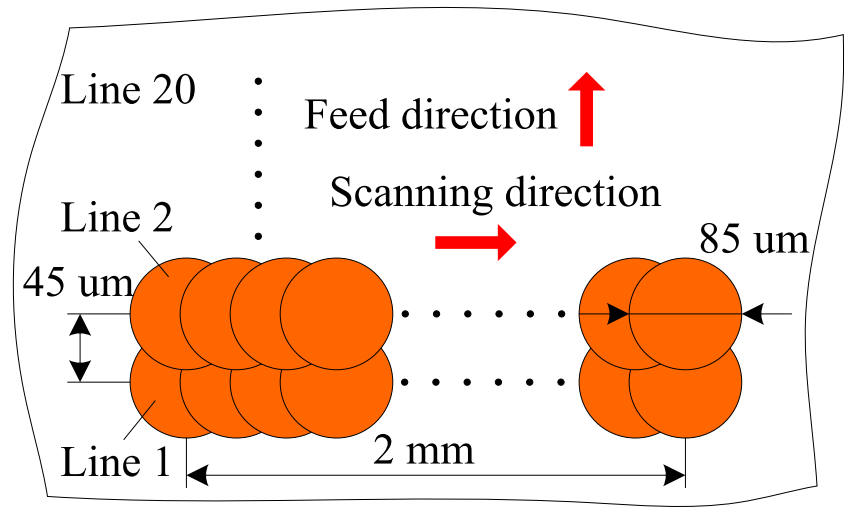

Fig. 1. Laser irradiation path. different tested cases listed in Table 1 . For each case, a total of 20 lines were irradiated.

The surface microstructure of the laser-irradiated regions was inspected by field-emission scanning electron microscopy (FE-SEM, JSM-7600F, JEOL, Japan), whereas the amorphous features of the sample before and after laser irradiation were characterized by Xray diffraction (XRD, D8 Discover, Bruker, Germany). Then, the irradiated MG surface was further polished with diamond abrasive paste to produce a mirror surface for subsequent nanoindentation experiments. Before nanoindentation, the amorphous features of the irradiated regions after polishing were characterized again by XRD. Nanoindentation tests were performed on an ENT-1100 nanoindentation instrument (Elionix Inc., Japan) using a Berkovich indenter and load-control mode. Three indentation loads (100, 400 , and $800 \mathrm{mN}$ ) were used and the corresponding loading/ unloading rates were set to 2,10 , and $10 \mathrm{mN} / \mathrm{s}$, respectively. The holding time at the maximum indentation load was $1 \mathrm{~s}$ for all nanoindentation tests. For each load, eight nanoindentation tests were carried out to ensure reliable results. After nanoindentation, the residual indents were inspected by FE-SEM.

\section{Results}

Fig. 2 shows the SEM morphologies of the laser-irradiated regions corresponding to the five cases investigated. Periodic microgrooves are observed for case 1, as shown in Fig. 2(a). The low scanning speed of $1 \mathrm{~mm} / \mathrm{s}$ results in large heat accumulation, promoting local temperature increase. Thus, many MG materials are heated and liquefied, which are further pushed to flow outward by the recoil pressure, forming microgrooves as well as surrounding protrusions by subsequent re-solidification. For cases 2 and 3, the laser pulse tracks are uniformly distributed on the irradiated regions because of the lower heat accumulation for higher scanning speeds. Along the scanning direction, it can be clearly seen that the previous laser pulse tracks are partially covered by the subsequent tracks, and the overlap width is dependent on the laser scanning speed. As shown in Fig. 2(b) and (c), when the scanning speed increases from $5 \mathrm{~mm} / \mathrm{s}$ (case 2) to $10 \mathrm{~mm} / \mathrm{s}$ (case 3), the overlap width decreases. For case 4 , although the average laser power is increased to $0.697 \mathrm{~W}$, the laser pulse tracks are still uniformly distributed on the irradiated region (as shown in Fig. 2(d)) owing to the high scanning speed of $10 \mathrm{~mm} / \mathrm{s}$, and the overlap width remains unchanged with respect to that observed for case 3 in Fig. 2(c). Turning to case 5 , after 10 cycles of laser irradiation, the irradiated region shows a similar laser track distribution to case 3 . This suggests that the liquefied MG materials re-solidify before each subsequent laser irradiation cycle, because of the small interaction volume and very high cooling rate during nanosecond pulsed laser irradiation. Thus, subsequent laser irradiation cycles merely repeat the first irradiation process, and the effect of heat accumulation in the previous cycles on the residual morphology is negligible.

Fig. 3 presents the XRD patterns obtained for the as-cast MG surface and laser-irradiated regions. The diffraction pattern of the as-cast MG surface is characterized by a typical broad halo without

Table 1

Experimental parameters corresponding to the five tested cases.

\begin{tabular}{llll}
\hline & $\begin{array}{l}\text { Average laser } \\
\text { power }(\mathrm{W})\end{array}$ & $\begin{array}{l}\text { Laser scanning } \\
\text { speed }(\mathrm{mm} / \mathrm{s})\end{array}$ & $\begin{array}{l}\text { Number of } \\
\text { irradiation cycle }\end{array}$ \\
\hline Case 1 & 0.422 & 1 & 1 \\
Case 2 & 0.422 & 5 & 1 \\
Case 3 & 0.422 & 10 & 1 \\
Case 4 & 0.697 & 10 & 1 \\
Case 5 & 0.422 & 10 & 10 \\
\hline
\end{tabular}




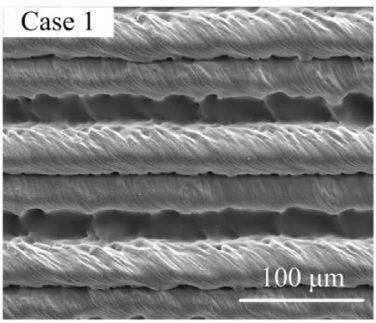

(a)

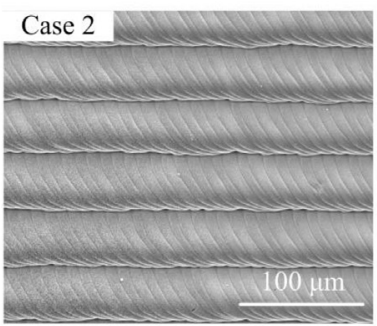

(b)

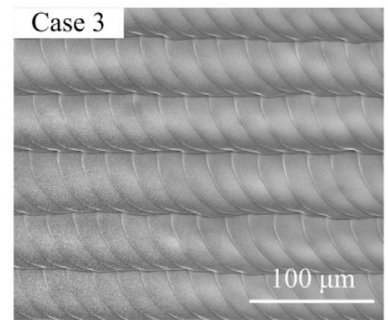

(c)

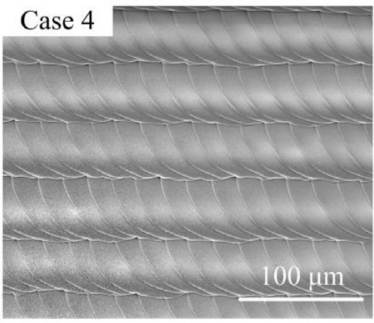

(d)

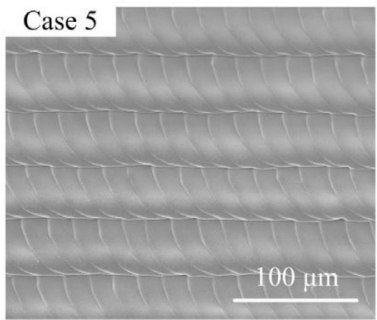

(e)

Fig. 2. SEM morphologies of the laser-irradiated regions corresponding to the five cases investigated: (a) 1, (b) 2 , (c) 3 , (d) 4 , and (e) 5 .

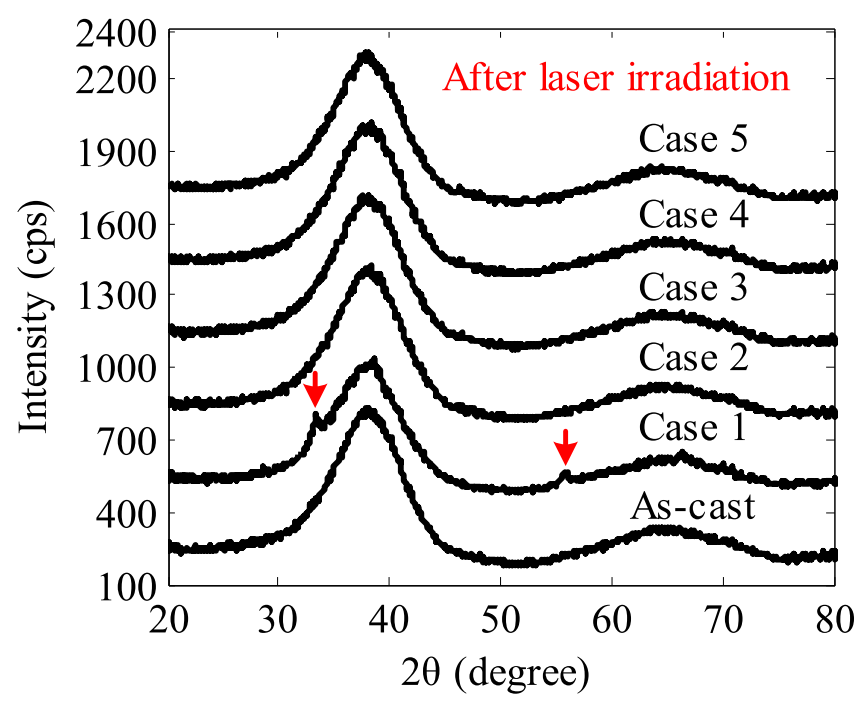

Fig. 3. XRD patterns measured for the as-cast MG surface and laser-irradiated regions.

any distinguishable sharp peaks, confirming the amorphous nature of this sample. For a same laser power of $0.422 \mathrm{~W}$, when the scanning speed is relatively high ( 5 and $10 \mathrm{~mm} / \mathrm{s}$ for cases 2 and 3 respectively), amorphous features emerge in the XRD patterns because of the very high heating and cooling rate during the pulsed laser-MG interaction. According to the thermal model in Ref. [30], when the MG surface was irradiated by a single laser pulse with an average power of $0.422 \mathrm{~W}$, the surface temperature varying with the time was calculated and illustrated in Fig. 4. It is noted that the surface temperature is increased very fast, and at about $19.7 \mathrm{~ns}$, it reaches the maximum value of $4380 \mathrm{~K}$. Accordingly, the average heating rate is over $10^{11} \mathrm{~K} / \mathrm{s}$, and then the surface temperature is decreased at a cooling rate being over $10^{10} \mathrm{~K} / \mathrm{s}$. The heating and cooling rates are greatly larger than the critical heating and cooling rates required for suppressing the crystallization of Vitreloy 1 (200 and $1 \mathrm{~K} / \mathrm{s}$ respectively) [31], and thus the irradiated surfaces remain amorphous characteristics. However, some weak crystalline peaks appear in the XRD pattern of case 1 . This is due to the large amount

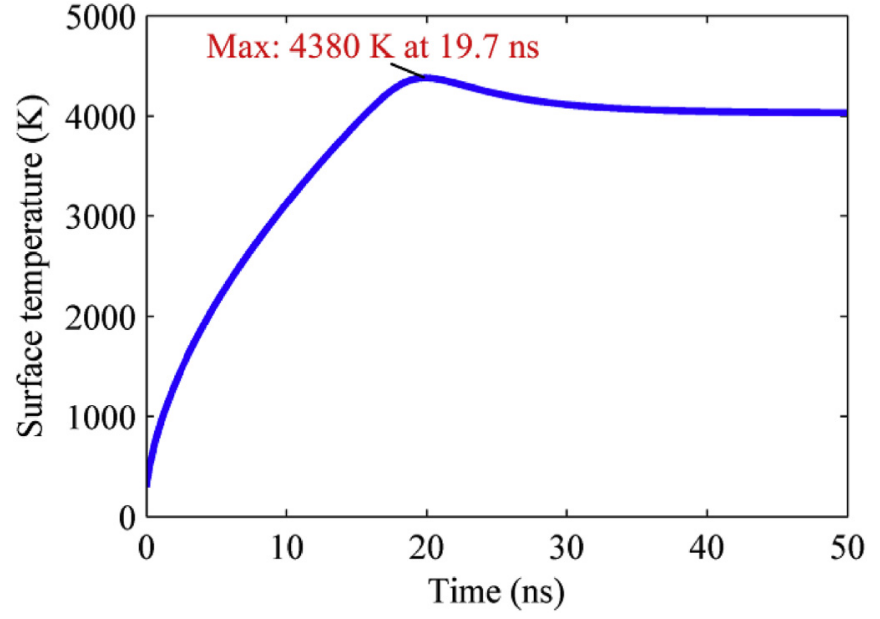

Fig. 4. The calculated surface temperature varying with the time when the MG surface was irradiated with a single laser pulse at the average laser power of $0.422 \mathrm{~W}$.

of heat accumulation at the relatively low scanning speed of $1 \mathrm{~mm} /$ $\mathrm{s}$, which greatly reduces the cooling rate during the laser pulse off, resulting in nucleation and growth of some crystalline phases. On the other hand, for cases 4 and 5, even though the average laser power is increased to $0.697 \mathrm{~W}$ or the number of irradiation cycle is increased to 10 , the irradiated regions still show amorphous characteristics because of the high scanning speed of $10 \mathrm{~mm} / \mathrm{s}$.

Fig. 5(a) shows the SEM morphology of the laser-irradiated region of case 1 after polishing. The wrinkled surfaces shown in Fig. 2(a) become smooth after polishing, and microgrooves are still observed between the two smooth surfaces. The width of a single smooth surface is about $70 \mu \mathrm{m}$, and nanoindentation tests were performed around the center of the smooth surface, as illustrated in Fig. 5(a). Fig. 5(b) compares the XRD patterns measured for the ascast MG surface and laser-irradiated regions after polishing. All XRD patterns show amorphous characteristics, indicating that the crystalline layer formed on the case 1 surface has been removed by the polishing treatment.

Fig. 6(a) compares the hardness of the as-cast MG surface and 


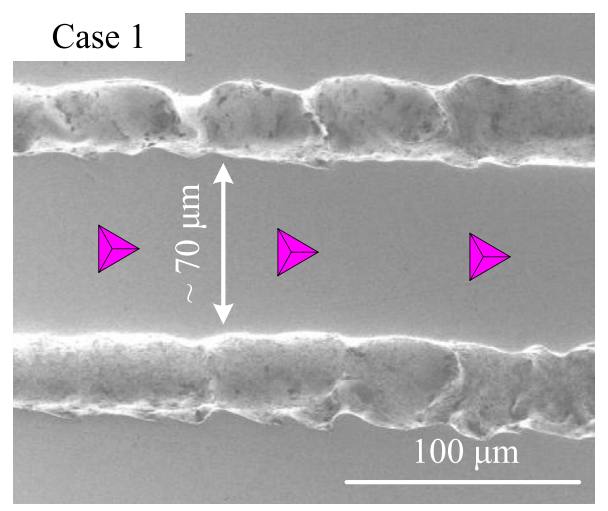

(a)

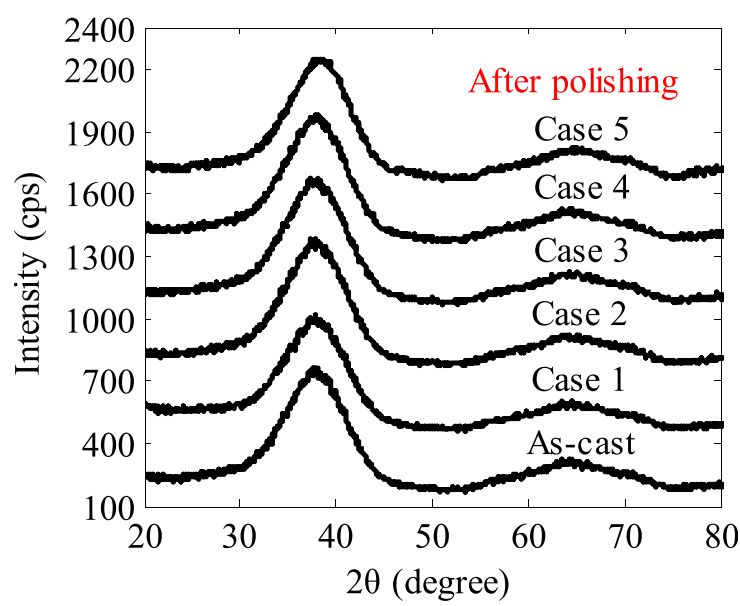

(b)

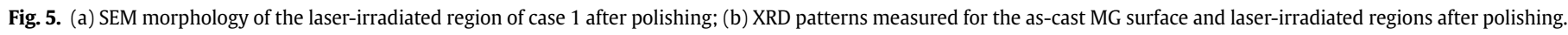

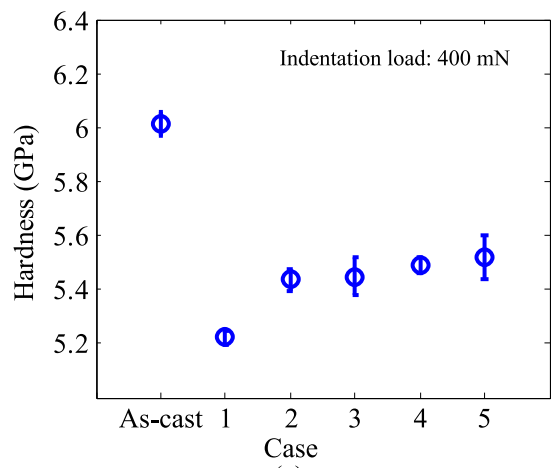

(a)

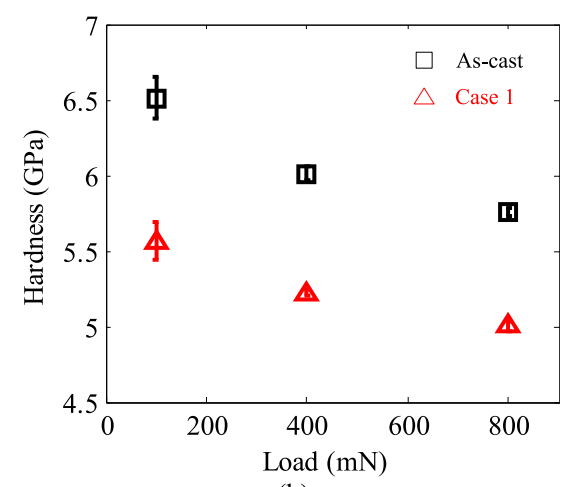

(b)

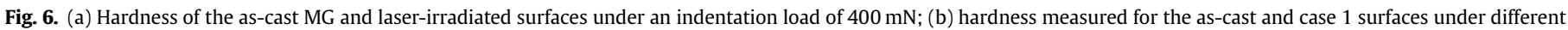
indentation loads.

laser-irradiated regions under an indentation load of $400 \mathrm{mN}$. The average hardness is $6.01 \mathrm{GPa}$ for the as-cast MG surface, decreases to $5.22 \mathrm{GPa}$ for case 1 , and then slightly recovers to $5.44,5.45,5.49$, and $5.52 \mathrm{GPa}$ for cases 2, 3, 4, and 5, respectively. Furthermore, for the same average laser power of $0.422 \mathrm{~W}$ (cases $1-3$ ), the low scanning speed of $1 \mathrm{~mm} / \mathrm{s}$ leads to the largest hardness decrease of $0.79 \mathrm{GPa}$, and the softening degree measured for laser scanning speeds of 5 and $10 \mathrm{~mm} / \mathrm{s}$ is almost the same. For the same average laser power of $0.422 \mathrm{~W}$ (cases 3 and 5), increasing the number of laser irradiation cycles from 1 to 10 results in a slight increase in hardness. For the same scanning speed of $10 \mathrm{~mm} / \mathrm{s}$ (cases 3 and 4), the slight increase in laser power also leads to a small increase in the hardness of the irradiated region. The comparison in Fig. 6(a) clearly indicates that nanosecond pulsed laser irradiation results in softening of the Zr-based MG for all five cases investigated, and this effect is more marked at lower scanning speeds.

Fig. 6(b) shows a comparison of the hardness of the as-cast MG and case 1 surfaces under different indentation loads (100, 400, and $800 \mathrm{mN}$ ). The indentation hardness decreases with increasing indentation load for both the as-cast MG and case 1 surfaces. This phenomenon is well known as indentation size effect [32]. Furthermore, under the three different indentation loads examined, the average hardness of the case 1 surface is much lower than that of the as-cast MG surface. The results in Fig. 6(b) further confirm the softening role of the nanosecond pulsed laser irradiation.
Fig. 7(a) illustrates typical load-depth curves obtained for the ascast MG and case 1 surfaces under the indentation load of $100 \mathrm{mN}$. For the same indentation load, the penetrated depth for the case 1 surface is $923 \mathrm{~nm}$, which is $68 \mathrm{~nm}$ larger than that of the as-cast surface $(855 \mathrm{~nm})$. The significant increase in penetrated depth further indicates the marked softening of case 1. Furthermore, discontinuous depth bursts, commonly referred to as "serrated flow" in the field of MGs, appear in the load-depth curves. The loaddepth curve corresponding to case 1 exhibits a more pronounced serrated flow, compared with that observed for the as-cast MG. This difference was further investigated using the depth difference method [33], and the results are presented in Fig. 7(b). For ease of comparison, the depth difference of case 1 is shifted by $4 \mathrm{~nm}$ along the vertical axis. A single serrated flow in the load-depth curve results in a sharp peak in the depth difference-load curve shown in Fig. 7(b). Furthermore, a serrated flow with large depth bursts results in a large depth difference. From Fig. 7(b), it is noted that much more sharp peaks with depth difference above $3 \mathrm{~nm}$, appear in the depth difference-load curve of case 1 compared with that of as-cast MG. For ease of comparison, the statistic results of the depth difference being over $3 \mathrm{~nm}$ in the load range of $10-100 \mathrm{mN}$ in Fig. 7(b) are listed in Table 2. Both the number and sum of the depth difference being over $3 \mathrm{~nm}$ for case 1 are greatly larger than those of as-cast MG. The results in Fig. 7 and Table 2 thus clearly indicate that nanosecond pulsed laser irradiation promotes the serrated flow behavior of MG during nanoindentation. 

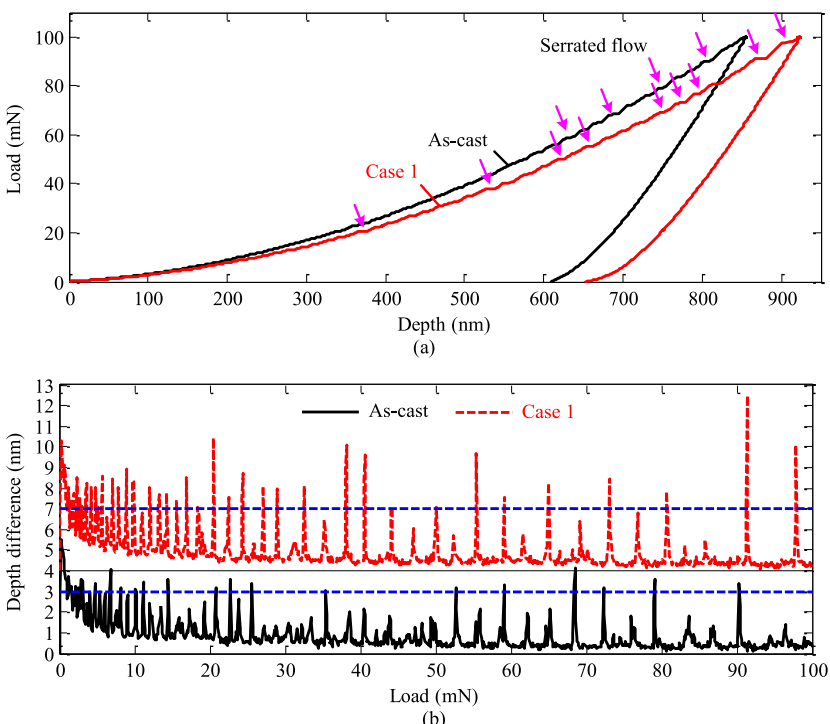

(b)

Fig. 7. (a) Load-depth curves and corresponding depth difference plots (b) obtained for the as-cast MG and case 1 surfaces under an indentation load of $100 \mathrm{mN}$. For ease of comparison, the depth difference of case 1 is shifted by $4 \mathrm{~nm}$ along the vertical axis.

Table 2

Statistic results of the depth difference being over $3 \mathrm{~nm}$ in the load range of $10-100 \mathrm{mN}$ in Fig. 7(b).

\begin{tabular}{lll}
\hline & As-cast & Case 1 \\
\hline Number of depth difference being over 3 $\mathrm{nm}$ & 13 & 23 \\
Sum of depth difference being over 3 $\mathrm{nm}(\mathrm{nm})$ & 44 & 103 \\
\hline
\end{tabular}

Fig. 8 displays the residual indent morphologies obtained for the as-cast MG and case 1 surfaces. To confirm the general validity of the results, the SEM morphologies are shown for two different indentation loads, 100 and $800 \mathrm{mN}$, and two residual indent morphologies are presented under each condition. For the as-cast MG surface, prominent shear bands are observed around the indent edges for both indentation loads. However, shear bands are nearly invisible on the top surface of case 1 . As shear banding is regarded as the main plastic deformation mechanism in MGs, the significant difference in the shear bands around the indent edges of the two cases suggests that the plastic deformation of MG has been significantly affected by laser irradiation. This can be further confirmed by analyzing the depth difference-load curves illustrated in Fig. 9, where serrated flows are more prominent for the case 1-5 surfaces compared with the as-cast surface. The corresponding statistic results of the depth difference being over $5 \mathrm{~nm}$ as listed in Table 3 further prove that for all laser irradiation cases, the number and sum of the depth difference being over $5 \mathrm{~nm}$ are significantly larger than those of as-cast MG. Furthermore, the variation tendency of the sum of depth difference in Table 3 is very similar to that of the hardness shown in Fig. 6(a), i.e., the larger the sum is, the lower the hardness will be.

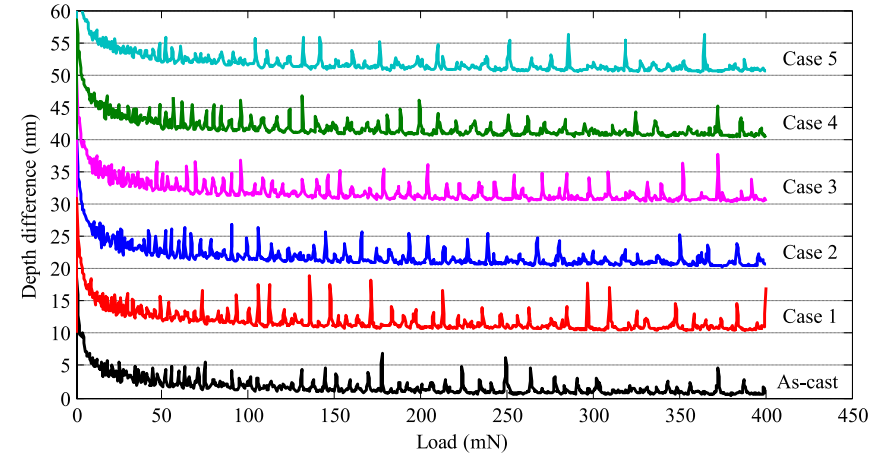

Fig. 9. Depth difference-load curves of as-cast and case 1-5 surfaces.

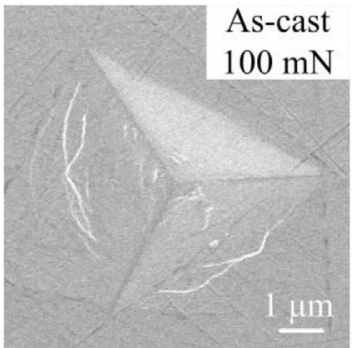

(a)

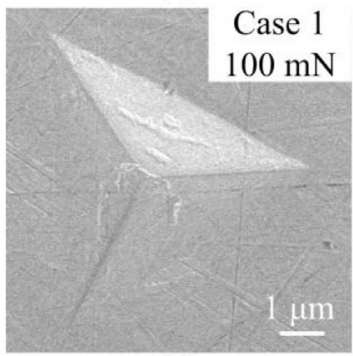

(e)

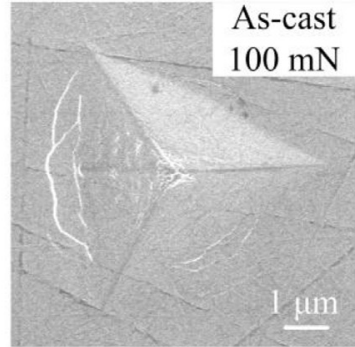

(b)

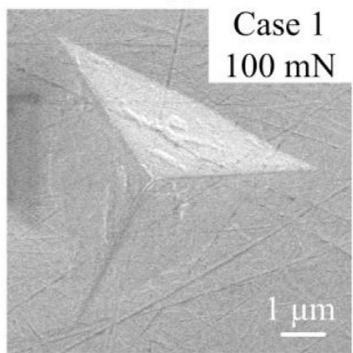

(f)

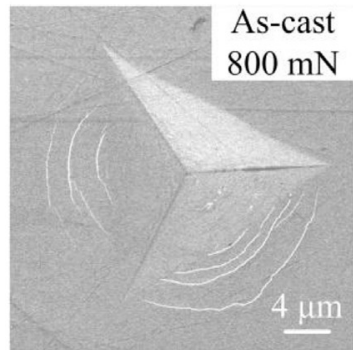

(c)

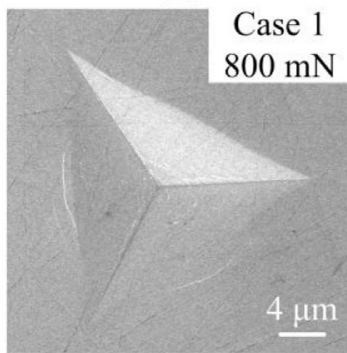

(g)

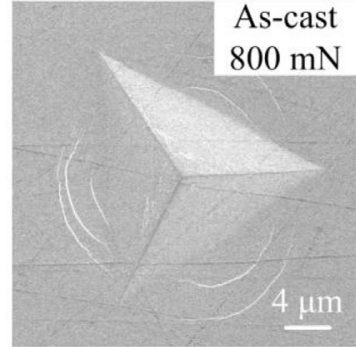

(d)

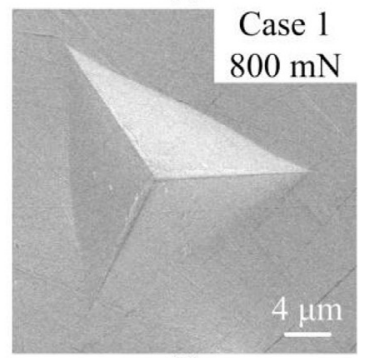

(h)

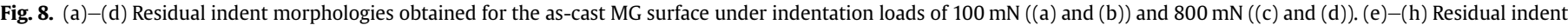

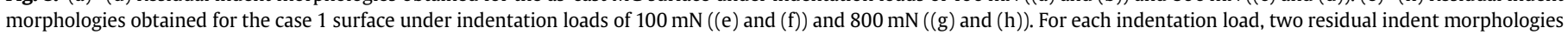
are displayed, to demonstrate the general validity of the results. 
Table 3

Statistic results of the depth difference being over $5 \mathrm{~nm}$ in the load range of $50-400 \mathrm{mN}$ in Fig. 9.

\begin{tabular}{|c|c|c|c|c|c|c|}
\hline & As-cast & Case 1 & Case 2 & Case 3 & Case 4 & Case 5 \\
\hline Number of depth difference being over $5 \mathrm{~nm}$ & 3 & 11 & 12 & 8 & 9 & 9 \\
\hline Sum of depth difference being over $5 \mathrm{~nm}(\mathrm{~nm})$ & 19 & 81 & 69 & 50 & 52 & 51 \\
\hline
\end{tabular}

\section{Discussion}

The results shown in Figs. 6-9, Tables 2 and 3 indicate that nanosecond pulsed laser irradiation softens the MG surface, markedly enhances the serrated flow but substantially suppresses the surface shear bands. Previous studies [34,35] reported that if the serrated flow was enhanced, more visible shear bands should appear around the indent edges. However, the opposite effect is observed for the laser-irradiated surface in the present study, for instance in case 1, as shown in Figs. 7 and 8. For this case, the enhanced serrated flow suggests more operations of shear bands, while the very few visible shear bands around the indent edges imply less nucleation and propagation of new shear bands on the top surface. A similar phenomenon has been reported in our previous study of pre-compression deformed MG [23], and was ascribed to the operation of pre-existing shear bands inside the sample. Recently, re-activation of pre-existing shear bands has been experimentally confirmed by an adjacent nanoindentation method [36], and the operation of these pre-existing shear bands could also promote the serrated flow.

Taking into account the laser-MG interaction, the explanation based on the re-activation of pre-existing shear bands inside the sample could also be adopted to rationalize the observed behavior of the laser-irradiated surface. Previous studies [20,37,38] reported that after laser shock peening of a MG coated by aluminum foil as absorption layer and immersed in water to limit the plasma expansion, large amounts of shear bands appeared on the MG surface. Fig. 10 presents an example showing the appearance of shear bands induced by laser shock peening obtained in Ref. [20] (Figure S2 in Supplementary information of Ref. [20]). Correspondingly, in the present study, as shown in Fig. 11, the removed layer of laser-irradiated surface by polishing can be regarded as the absorption layer, playing a similar role to the aluminum foil, while the argon shield gas has the function of limiting the plasma expansion. Accordingly, due to the shock pressure, pre-existing shear bands could have been generated in the subsurface layer of

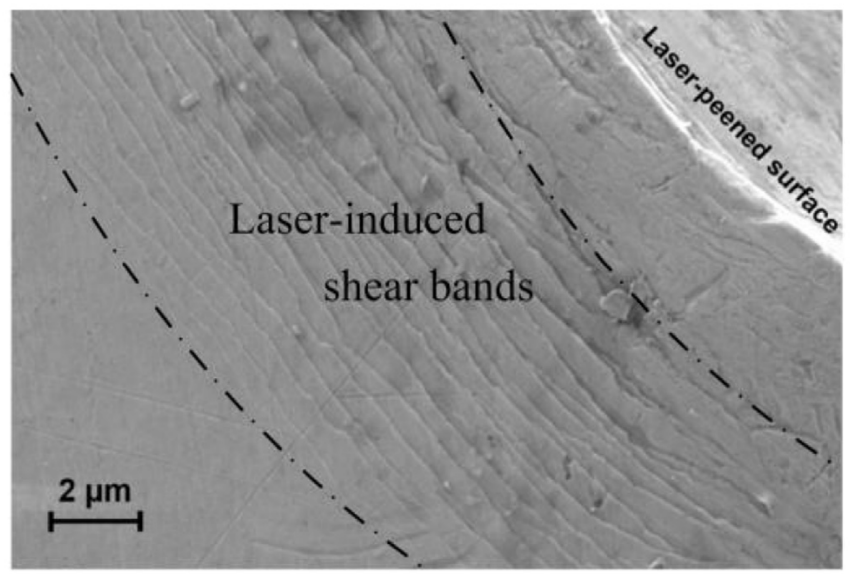

Fig. 10. An example showing the appearance of shear bands induced by laser shock peening obtained in Ref. [20].

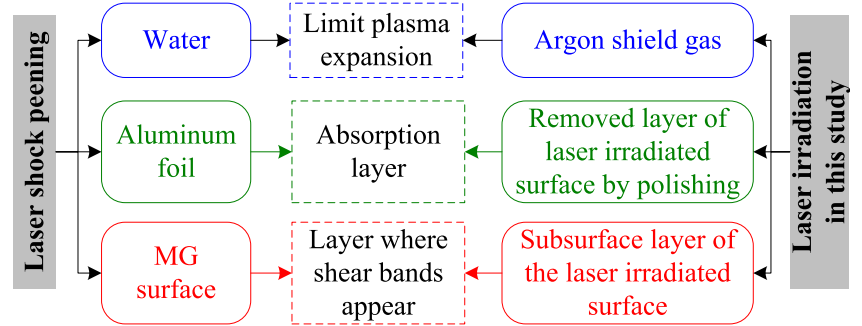

Fig. 11. The corresponding relationship between the laser shock peening and laser irradiation in this study.

the laser-irradiated surface subjected to the nanoindentation tests, even though they are not visible in the SEM image shown in Fig. 5(a). For case 1, as only very few shear bands are present on the top surface, it can be concluded that the enhanced serrated flow is caused by the operation of pre-existing shear bands inside the sample. Compared with the MG matrix, pre-existing shear bands are relatively soft regions [21]. During nanoindentation, the applied plastic strain is preferentially accommodated by propagation of these pre-existing shear bands, while the nucleation of new shear bands on the top surface is substantially suppressed. Therefore, the indentation hardness decreases for case 1, compared with that of the as-cast MG. Furthermore, a more prominent serrated flow can be observed for case 1 , for which only very few shear bands are visible on the top surface. For the same average laser power of $0.422 \mathrm{~W}$, when the laser scanning speed is increased to 5 and $10 \mathrm{~mm} / \mathrm{s}$ (cases 2 and 3), the overlap width along the scanning direction decreases, as shown in Fig. 2(b) and (c), i.e., the number of laser pulses for the same irradiation region decreases. Thus, the number of pre-existing shear bands for cases 2 and 3 decreases, and the indentation hardness is slightly recovered. For the same scanning speed of $10 \mathrm{~mm} / \mathrm{s}$ (cases 3 and 4), the small increase in indentation hardness for higher average laser power may result from more significant thermal effects [39-41], which slightly reduce the plastic flow ability of the MG. Moreover, when the number of laser irradiation cycles increases from 1 (case 3 ) to 10 (case 5$)$ at the same average laser power $(0.422 \mathrm{~W})$ and scanning speed $(10 \mathrm{~mm} / \mathrm{s})$, the accumulated heat effect in the repeated laser irradiation cycles may also result in a slight increase in indentation hardness, although no significant differences emerge between the surface morphologies shown in Fig. 2.

\section{Conclusions}

The mechanical properties of the nanosecond pulsed laserirradiated surfaces of Zr-based metallic glass (MG) were compared with those of the as-cast MG surface using nanoindentation. The results of the experiments indicate that nanosecond pulsed laser irradiation softened the irradiated regions; the effect of the irradiation showed a slight dependence on the laser irradiation parameters, and especially the laser scanning speed. Under an average laser power of $0.422 \mathrm{~W}$ and a scanning speed of $1 \mathrm{~mm} / \mathrm{s}$, the indentation hardness decreased from 6.01 GPa for the as-cast MG to $5.22 \mathrm{GPa}$ for the irradiated surface. Furthermore, compared with the results obtained for the as-cast surface, the 
load-depth curve obtained for the irradiated surface exhibited enhanced serrated flows, but fewer shear bands were visible on the top surface of the corresponding residual indents.

\section{Acknowledgments}

This work was supported by the National Natural Science Foundation of China (Grant No. 51705197, 11522221), Opening fund of State Key Laboratory of Nonlinear Mechanics (Grant No. LNM201802), Grant-in-Aid for JSPS Fellows (Grant No. 26-04048), Young Talent Lift Project of China Association for Science and Technology and Chinese Mechanical Engineering Society (CMES) (2017-2019), and the Fundamental Research Funds for the Central Universities.

\section{References}

[1] Y.B. Wang, D.D. Qu, X.H. Wang, Y. Cao, X.Z. Liao, M. Kawasaki, S.P. Ringer, Z.W. Shan, T.G. Langdon, J. Shen, Introducing a strain-hardening capability to improve the ductility of bulk metallic glasses via severe plastic deformation, Acta Mater. 60 (2012) 253-260.

[2] C.A. Schuh, T.C. Hufnagel, U. Ramamurty, Overview No.144-Mechanical behavior of amorphous alloys, Acta Mater. 55 (2007) 4067-4109.

[3] Y.H. Liu, G. Wang, R.J. Wang, D.Q. Zhao, M.X. Pan, W.H. Wang, Super plastic bulk metallic glasses at room temperature, Science 315 (2007) 1385-1388.

[4] L.C. Zhang, F. Jiang, D.H. Zhang, L. He, J. Sun, J.T. Fan, Z.F. Zhang, In-Situ precipitated nanocrystal beneficial to enhanced plasticity of $\mathrm{Cu}-\mathrm{Zr}$ based bulk metallic glasses, Adv. Eng. Mater. 10 (2008) 943-950.

[5] G. Chen, H. Bei, Y. Cao, A. Gali, C.T. Liu, E.P. George, Enhanced plasticity in a Zrbased bulk metallic glass composite with in situ formed intermetallic phases, Appl. Phys. Lett. 95 (2009), 081908.

[6] J.W. Qiao, Y. Zhang, G.L. Chen, Fabrication and mechanical characterization of a series of plastic Zr-based bulk metallic glass matrix composites, Mater. Des. 30 (2009) 3966-3971.

[7] G.J. Wu, R. Li, Z.Q. Liu, B.Q. Chen, Y. Li, Y. Cai, T. Zhang, Induced multiple heterogeneities and related plastic improvement by laser surface treatment in CuZr-based bulk metallic glass, Intermetallics 24 (2012) 50-55.

[8] J. Gu, M. Song, S. Ni, X.Z. Liao, S.F. Guo, Improving the plasticity of bulk metallic glasses via pre-compression below the yield stress, Mater. Sci. Eng., A 602 (2014) 68-76.

[9] F. Qiu, P. Shen, T. Liu, Q.C. Jiang, Enhanced ductility in a Zr65Cu15Al10Ni10 bulk metallic glass by nanocrystallization during compression, Mater. Des. 36 (2012) 168-171.

[10] H.B. Yu, J. Hu, X.X. Xia, B.A. Sun, X.X. Li, W.H. Wang, H.Y. Bai, Stress-induced structural inhomogeneity and plasticity of bulk metallic glasses, Scripta Mater. 61 (2009) 640-643.

[11] Y. Zhang, W.H. Wang, A.L. Greer, Making metallic glasses plastic by control of residual stress, Nat. Mater. 5 (2006) 857-860.

[12] B.Q. Chen, S.J. Pang, P.P. Han, Y. Li, A.R. Yavari, G. Vaughan, T. Zhang Improvement in mechanical properties of a $\mathrm{Zr}$-based bulk metallic glass by laser surface treatment, J. Alloys Compd. 504 (2010) S45-S47.

[13] B.Q. Chen, Y. Li, M. Yi, R. Li, S.J. Pang, H. Wang, T. Zhang, Optimization of mechanical properties of bulk metallic glasses by residual stress adjustment using laser surface melting, Scripta Mater. 66 (2012) 1057-1060.

[14] Y.H. Zhu, J. Fu, C. Zheng, Z. Ji, Effect of laser shock peening without absorbent coating on the mechanical properties of $\mathrm{Zr}$-based bulk metallic glass, Optic Laser. Technol. 75 (2015) 157-163.

[15] J. Fu, Y.H. Zhu, C. Zheng, R. Liu, Z. Ji, Enhanced plasticity of bulk metallic glass in different aspect ratios via laser shock peening with multiple impacts, Optic Laser. Technol. 83 (2016) 43-48.

[16] J. Fu, Y.H. Zhu, C. Zheng, R. Liu, Z. Ji, Effect of laser shock peening on mechanical properties of Zr-based bulk metallic glass, Appl. Surf. Sci. 313 (2014) 692-697.

[17] J. Fu, Y.H. Zhu, C. Zheng, R. Liu, Z. Ji, Effect of laser shock peening on the compressive deformation and plastic behavior of $\mathrm{Zr}$-based bulk metallic glass, Optic Laser. Eng. 86 (2016) 53-61.

[18] Y.H. Zhu, J. Fu, C. Zheng, Z. Ji, Structural and mechanical modifications induced on Zr-based bulk metallic glass by laser shock peening, Optic Laser. Technol. 86 (2016) 54-60.

[19] J. Fu, Y.H. Zhu, C. Zherig, R. Liu, Z. Ji, Evaluate the effect of laser shock peening on plasticity of Zr-based bulk metallic glass, Optic Laser. Technol. 73 (2015) 94-100.

[20] Y.F. Cao, X. Xie, J. Antonaglia, B. Winiarski, G. Wang, Y.C. Shin, P.J. Withers, K.A. Dahmen, P.K. Liaw, Laser shock peening on Zr-based bulk metallic glass and its effect on plasticity: experiment and modeling, Sci. Rep. 5 (2015) 10789.

[21] R. Maass, K. Samwer, W. Arnold, C.A. Volkert, A single shear band in a metallic glass: local core and wide soft zone, Appl. Phys. Lett. 105 (2014), 171902.

[22] C.A. Schuh, T.G. Nieh, A survey of instrumented indentation studies on metallic glasses, J. Mater. Res. 19 (2004) 46-57.

[23] H. Huang, J.L. Zhang, C.H. Shek, J.W. Yan, Effects of pre-compression deformation on nanoindentation response of Zr65Cu15Al10Ni10 bulk metallic glass, J. Alloys Compd. 674 (2016) 223-228.

[24] F.C. Li, J. Gu, M. Song, S. Ni, S.F. Guo, The evolution of local mechanical properties of bulk metallic glasses caused by structural inhomogeneity, J. Alloys Compd. 591 (2014) 315-319.

[25] F.C. Li, M. Song, S. Ni, S.F. Guo, X.Z. Liao, Correlation between hardness and shear banding of metallic glasses under nanoindentation, Mater. Sci. Eng., A 657 (2016) 38-42.

[26] H. Huang, J.W. Yan, On the surface characteristics of a $\mathrm{Zr}$-based bulk metallic glass processed by microelectrical discharge machining, Appl. Surf. Sci. 355 (2015) 1306-1315.

[27] H. Huang, J.W. Yan, Microstructural changes of Zr-based metallic glass during microelectrical discharge machining and grinding by a sintered diamond tool, J. Alloys Compd. 688 (2016) 14-21.

[28] H. Huang, J. Noguchi, J.W. Yan, Shield gas induced cracks during nanosecondpulsed laser irradiation of Zr-based metallic glass, Appl. Phys. A. Mater. 122 (2016) 881.

[29] H. Huang, J.W. Yan, Surface patterning of Zr-based metallic glass by laser irradiation induced selective thermoplastic extrusion in nitrogen gas, J. Micromech. Microeng. 27 (2017), 075007.

[30] M.Q. Jiang, Y.P. Wei, G. Wilde, L.H. Dai, Explosive boiling of a metallic glass superheated by nanosecond pulse laser ablation, Appl. Phys. Lett. 106 (2015), 021904.

[31] J. Schroers, A. Masuhr, W.L. Johnson, R. Busch, Pronounced asymmetry in the crystallization behavior during constant heating and cooling of a bulk metallic glass-forming liquid, Phys. Rev. B 60 (1999) 11855-11858.

[32] M.C. Li, M.Q. Jiang, F. Jiang, L. He, J. Sun, Testing effects on hardness of a Zrbased metallic glass under nanoindentation, Scripta Mater. 138 (2017) $120-123$.

[33] H. Huang, H.W. Zhao, Z.Y. Zhang, Z.J. Yang, Z.C. Ma, Influences of sample preparation on nanoindentation behavior of a $\mathrm{Zr}$-based bulk metallic glass, Materials 5 (2012) 1033-1039.

[34] Y.I. Golovin, V.I. Ivolgin, V.A. Khonik, K. Kitagawa, A.I. Tyurin, Serrated plastic flow during nanoindentation of a bulk metallic glass, Scripta Mater. 45 (2001) 947-952.

[35] H. Huang, H. Zhao, Indenter geometry affecting indentation behaviors of the Zr-based bulk metallic glass, Mater. Trans. 55 (2014) 1400-1404.

[36] H. Huang, J.W. Yan, Investigating shear band interaction in metallic glasses by adjacent nanoindentation, Mater. Sci. Eng., A 704 (2017) 375-385.

[37] L. Wang, L. Wang, Y.F. Xue, H.F. Zhang, H.M. Fu, Nanoindentation response of laser shock peened Ti-based bulk metallic glass, AIP Adv. 5 (2015), 057156.

[38] Y.P. Wei, B.C. Wei, W. Xi, G.Y. Xu, L. Li, X.Q. Wu, H.W. Song, C.G. Huang, A novel micro-scale plastic deformation feature on a bulk metallic glass surface under laser shock peening, Chin. Phys. Lett. 30 (2013), 036201.

[39] S. Xie, E.P. George, Hardness and shear band evolution in bulk metallic glasses after plastic deformation and annealing, Acta Mater. 56 (2008) 5202-5213.

[40] P. Murali, U. Ramamurty, Embrittlement of a bulk metallic glass due to sub-Tg annealing, Acta Mater. 53 (2005) 1467-1478.

[41] J. Gu, M. Song, S. Ni, S.F. Guo, Y.H. He, Effects of annealing on the hardness and elastic modulus of a Cu36Zr48Al8Ag8 bulk metallic glass, Mater. Des. 47 (2013) 706-710. 